'The learned author is, I think, altogether wrong in imagining that the dryness of adjacent bodies can have any ill effect. In common with the great mass of electricians of this time, as well as his contemporaries, he has overlooked a real cause of deterioration. I allude to the imperfect conducting power of cushions, made as they are usually, of silk, or leather stuffed with hair, or other nonconducting substances. The desiccation of the cushion and other parts of the rubber, may counteract the benefit otherwise produced by any increase of aridity in the surrounding medium.

By stuffing the cushions with the elastic iron shreds scraped off from weaver's reeds in manufacturing them, and making a communication between the shreds and the steel spring supporting the cushion and attached to the negative conductor, I have seen the sparks yielded by a machine more than trebled in length, and frequency.

As a coating for the cushion, upon the whole, I find the aurum musivum, more efficacious than the amalgam usually employed, which is apt to adhere to the glass, and promote the passage of sparks from the cushion to the collecting points of the positive conductor. I question if the amalgam does not owe its efficacy to its conducting power, which tends to compensate the absence of this property in the cushion.

In speaking of experiments performed by means of electrical ma. chines, the poles and conductors may in general be treated as synonimous; yet strictly the poles are those parts of the conductors, or conducting surfaces in connexion with them, between which the discharge takes place; so that when insulated metallic rods, however long, are each at one end in contact with the conductors of the machine, the poles may be at the other ends of the rods. This view of the subject is generally recognised in the case of Voltaic series, which not being terminated by conductors, in the technical sense used in speaking of the machine, gives rise, in this respect, to less cause of misapprehension.

I conceive it an error to suppose that the association of a large conductor with a machine contributes to the intensity of the sparks. It appears to me to render the sparks shorter, and less frequent, though otherwise larger.

\title{
Notice of the application of the process of transferring to the art of Die Sinking.
}

By Frankux Prace, Lecturer on Machines in the Franklin Institute.

TO THE COMMITTEE ON PURMTCATYONS.

Gentlemen,--Several years since it was desirable in the management of the Philadelphia Museum, to be possessed of appropriate medals; for the accomplishment of this object, dies, the device of which was a portrait of Charles W. Peale, were executed in a most satisfactory manner by Mr. C. Gobrecht. One of these dies was unfortunately cracked in the hardening, and thus rendered useless. It subsequently became still more desirable that this object should 
be carried into execution, and that the likeness of the founder of the museum should be preserved. A prosecution of the subject led to the results which I will now state.

In the spring of $1825, \mathrm{Mr}$. M. W. Baldwin, aware of the applicability of the process of transferring, to die sinking for coinage, made at that time a transfer press, with which Mr. Kneass, of the United States Mint, made a perfectly successful experiment in the workshop of the former.

The object above stated led to a revival of this process, and its prosecution to completion, and $I$ have now the pleasure to present to the Institute one of those medals, stuck in silver, from dies which were made in the fall of 1832 by transfer, from the originals engraved by $\mathrm{Mr}$. Gobrecht. I do not offer this medal as a sample of workmanship in this department of the arts: the first specimen in any art is generally, from that circumstance alone, inferior; but it is offered as a creditable specimen, and at the same time a satisfactory dernonstration that the process of transferring is applicable to die sinking, with advantages that may be stated in a few words. The first of these advantages is great economy of labour. Any required number of dies may be taken from the original roller, all of them being fac similes of the original die. The process exercises, also, an advantageous effect on the metal, in raising it gently, and by successive efforts, with an equal condensation of the particles throughout all the portions of its surface, admitting at the same time of as many annealings as the relief of the device may require; of which the limits, as far as I can judge from my experience, are beyond the requisite elevation. From these facts I am fully authorized in believing that the risk of loss of the nearly finished work by cracking, in hardening, is much reduced.

A brief notice of the process, (which is applicable to all kinds of dies,) for general information, is all that I need offer, as an intimate knowledge could only be acquired by conducting it.

The die is engraved in the usual manner, except that it has a greater width of margin. It is then hardened and placed in the press, in contact with a soft roll, and subjected to the necessary pressure and roller, with occasional annealings, as may be found necessary, the frequency of which will depend on the depth of the device. The roll is then hadened, and the operation repeated with the substitution of a soft die, to receive the impression from the hardened roll; the die is then prepared and hardened in the usual manner; the roll being preserved to repeat the transfers, as often as may be desired.

The process of transfer is now used in the engraving department of the U. S. Mint, under the direction of $\mathrm{Mr}$. W $\mathrm{m}$. Kneass, to whose liberal aid I am much indebted in the prosecution of this process to its successful completion.

I have the honour to be, Very respectfully, yours, Franiklin Pealf.

Philadelphia, April 24, 1832. 